\title{
Pseudomyxoma Peritonei from a Borderline Mucinous Tumor Arising in an Ovarian Mature Cystic Teratoma: A Rare Case Report
}

Chiruvella $A^{1}$, Staley $C^{1}{ }^{1}$, Khanna $\mathbf{N}^{2}$, Russell $\mathbf{M}^{1}$, Maithel $\mathbf{S K}^{1}$, Adsay $\mathbf{V}^{3}$, Horowitz $\mathbf{I R}^{2}$, Staley $\mathbf{C}^{1}$ and Winer $\mathbf{J}^{1^{*}}$

${ }^{1}$ Division of Surgical Oncology, Emory University, Atlanta, GA, USA

${ }^{2}$ Division of Gynecologic Oncology, Emory University, Atlanta, GA, USA

${ }^{3}$ Division of Pathology, Emory University, Atlanta, GA, USA

"Corresponding author: Winer J, Division of Surgical Oncology, Emory University, Atlanta, GA, USA, Tel: 4047788059; E-mail: jhwiner@emory.edu

Received date: April 06, 2016; Accepted date: June 10, 2016; Published date: June 17, 2016

Copyright: $\odot 2016$ Chiruvella A, et al. This is an open-access article distributed under the terms of the Creative Commons Attribution License, which permits unrestricted use, distribution, and reproduction in any medium, provided the original author and source are credited.

\begin{abstract}
Pseudomyxoma peritonei (PMP) is a rare associated diagnosis of peritoneal carcinomatosis which, classically, has been characterized by a slowly progressive disease process. More recently, Ronnett et al., have histologically classified PMP into the slow growing disseminated peritoneal adenomucinosis (DPAM), the much more aggressive and invasive peritoneal mucinous carcinomatosis (PMCA) and an intermediate group (ID). Recent studies have shown that most cases of PMP arise from ruptured appendiceal tumors with dissemination of mucin producing epithelial cells into the peritoneal cavity. PMP, contrary to popular belief, almost never arises from a ruptured primary ovarian mucinous tumor, corroborated by two of the largest series on ruptured borderline mucinous ovarian neoplasms. A small number of cases have been reported, however, with peritoneal carcinomatosis secondary to rupture of an ovarian mucinous tumor associated with a mature cystic teratoma. These are usually CK7 negative and CK20 positive, and are immunohistochemically consistent with intestinal type of mucinous tumors that arise from intestinal elements of the teratoma. Other malignancies such as squamous cell carcinomas, malignant endodermal sinus tumors and chorio-carcinomas have been shown to arise from a mature teratoma. We present a case report describing one such rare case of DPAM arising from a ruptured borderline mucinous ovarian tumor in the background of a cystic teratoma.
\end{abstract}

Keywords: Tumor; Disease; Mucin; Carcinoma

\section{Introduction}

PMP is an uncommon entity with an incidence of 1-2 per million cases per year [1]. Many cases are found unexpectedly at laparotomy or laparoscopy, or may be suspected at cross sectional imaging during staging of another pathological entity. Werth in 1884 coined the term PMP, initially describing it in association with a mucinous tumor of the ovary. In 1901, Frankel described a case associated with a mucinous appendiceal cyst. Over the years, the term PMP has been used to describe almost every diffuse peritoneal mucinous tumor including those caused by lower GI tract and appendiceal mucinous primaries. The term PMP has been applied to carcinomatosis originating from the colon, rectum, stomach, gall bladder, small intestine, urinary bladder, lung, breast, fallopian tube and pancreas although the classic definition is from appendiceal or colonic tumors $[2,3]$.

Controversy persists over the classification of mucinous epithelial neoplasms and their relation to PMP. Several recent studies, including those by Ronnett et al., have redefined PMP as a specific clinicpathologic syndrome in which mucinous ascites is associated with peritoneal lesions characterized by histologically bland to low grade adenomatous mucinous epithelium, along with pools of extracellular mucin and fibrosis originating from appendiceal tumors [4-6]. Ronnett and colleagues, coined new terminology forgoing PMP altogether and reported a pathologic system that has become widely accepted in the literature [6]. They classified low grade tumors with bland peritoneal mucinous neoplastic epithelium as DPAM/disseminated peritoneal adenomucinosis and high grade tumors with mucinous epithelial architecture similar to that of an invasive cancer, as PMCA/peritoneal mucinous carcinomatosis. They also included an intermediate group demonstrating a mixture of DPAM and PMCA that was termed PMCA-I. This classification is important from a prognostic standpoint, as patients with DPAM appear to obtain maximum survival benefit from cytoreductive surgery and heated intraperitoneal chemotherapy (CRS and HIPEC) while those with PMCA behave more like peritoneal carcinomatosis of lower GI origin.

Multiple studies have provided morphologic, immunohistochemical and molecular evidence that PMP/DPAM is almost always derived from ruptured low grade adenomatous mucinous tumors of the appendix in both males and females and those primary ovarian mucinous tumors are almost never a source of PMP/DPAM [7-11]. Similarly, studies looking at ruptured primary ovarian mucinous tumors have not shown any association with PMP [8,12-14]. This has led to the current conundrum: whether primary mucinous ovarian tumors are truly associated with PMP/DPAM.

Aside from primary mucinous ovarian tumors, mucinous epithelial neoplasms associated with ovarian mature cystic teratomas are well documented in the literature. Most information regarding these tumors, is based on individual case reports and case series. Ronnett and Siedeman in 2003 reported a subset of mucinous epithelial tumors arising in a background of mature teratomas with an immunophenotype more suggestive of a gastrointestinal lineage than ovarian epithelial origin, and described an association with PMP in some cases [15]. Since then, several new case reports have been described with similar pathology. This paper describes one such case encountered at Emory University. 


\section{Case Presentation}

The patient is a 61 year old female who presented to an outside hospital in February 2014 with progressive shortness of breath and abdominal distension for 3 months. A CT scan of her abdomen and pelvis showed massive ascites along with a left ovarian/pelvic complex cystic mass and an incidental $3.3 \mathrm{~cm}$ left adrenal adenoma. Tumor markers were drawn as part of her workup, and these showed an elevated CEA of 80.55 and CA 125 of 75 with normal CA19-9 and LDH levels.

She underwent a paracentesis with aspiration of thick mucin concerning for PMP. An esophagogastroduodenoscopy (EGD) and colonoscopy were normal other than 15-20 shallow antral erosions. She was taken to the OR on 07/31/2014 and underwent an exploratory laparotomy with drainage of 15 liters of mucinous ascites and a left partial oophorectomy for biopsy. Pathology revealed pools of mucin with low cellularity and a mucinous cystadenoma of the ovary.

She was referred to Gynecologic and Surgical oncology at Emory where a repeat CT scan in 09/2014 showed reaccumulation of large volume ascites and a $20 \times 16 \mathrm{~cm}$ multiloculated complex cystic pelvic mass which was concerning for an immature teratoma. She was taken to the operating room on 10/22/2014 and underwent cytoreductive surgery and HIPEC that included omentectomy, hysterectomy with bilateral salpingo-oophorectomy, ileocolectomy, peritoneal stripping, hepatic capsulectomy, cholecystectomy, distal pancreatectomy and splenectomy, bilateral ureterolysis, complex left diaphragm repair, stamm gastrojejunal feeding tube placement, bilateral chest tube placement followed by intra-peritoneal chemotherapy with mitomycin $\mathrm{C}\left(30 \mathrm{mg} / \mathrm{m}^{2}\right.$ at time zero and additional $10 \mathrm{mg} / \mathrm{m}^{2}$ added to circuit at 60 minutes for a total perfusion of 90 minutes). Her calculated PCI (peritoneal carcinomatosis index) intraoperatively was 36 and she underwent a CC-0/1 (completeness of cytoreduction score) resection.

Her final pathology report showed a mucinous borderline tumor of the ovary with intestinal differentiation arising in association with a mature cystic teratoma. Ascitic fluid analysis showed extra-ovarian mucin with scant epithelium consistent with DPAM (Figure 1). There was no evidence of an invasive carcinoma, and a sclerosed and rudimentary appendix was identified with no evidence of an appendiceal tumor.

Her post-operative course was uneventful and she was discharged in 2 weeks, tolerating a regular diet. Follow up imaging has demonstrated no recurrence, with most recent CT scan in January 2016 showing no evidence of recurrent disease or ascites.

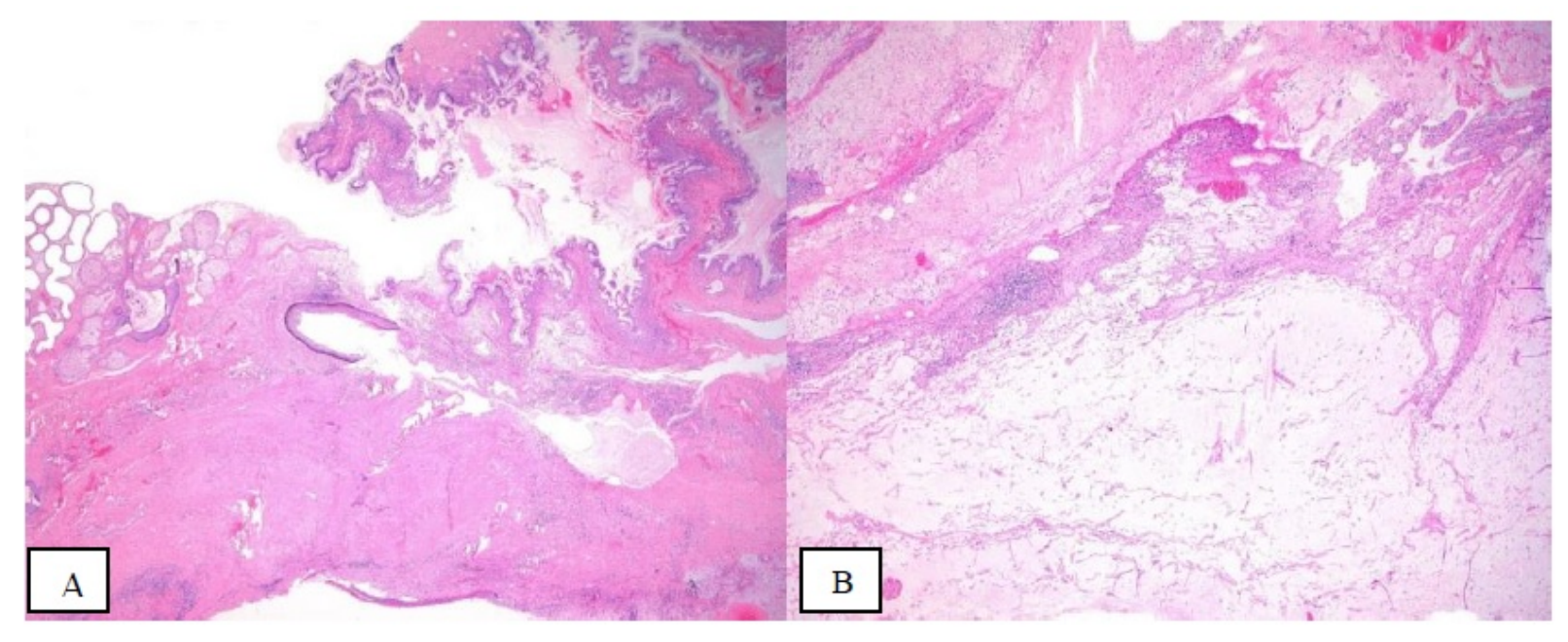

Figure 1: A) Teratoma represented as epidermal tissue and a mixture of sabecous and eccrine type glands (left and mid) transitions into mucinous cystic neoplasm with tall columnar mucinous cells. B) Peritoneal biopsy shows abundant acellular mucin (pale material, mostly in the lower aspect) admixed with stromal tissue showing some inflammatory infiltrates.

\section{Discussion}

Numerous cases involving PMP/DPAM have been published where an appendiceal mucinous tumor has not been identified, and some of these have occurred in women with mucinous tumors involving the ovaries, leading to a questionable conclusion of the ovary being the primary site of origin. However, morphologic, immunohistochemical and molecular genetic studies provide compelling evidence that the clinical syndrome of PMP is derived from ruptured low grade adenomatous mucinous tumors of the appendix even in women. Interestingly, studies of the more aggressive PMCA also showed no primary ovarian origin [4-6].

There are outliers to the above dogma. Ronnett et al. published three cases of mucinous tumors arising in an ovarian mature cystic teratoma that were associated with PMP. Two of these ovarian mucinous tumors had the same histologic features as those encountered in typical cases of PMP/DPAM derived from ruptured appendiceal mucinous tumors, with irregularly distributed bland to low-grade adenomatous mucinous epithelium associated with pseudomyxoma ovarii. The third tumor had histologic features of an adenoma combined with carcinoma, similar to cases of PMCA-I. In all three cases, the appendices were entirely normal microscopically, hence it was clear that the primary source was the mucinous tumor arising from a mature teratoma. Upon further examination with immuno-histochemical analysis, all three tumors displayed a lower GI tract phenotype rather than a primary ovarian mucinous tumor phenotype. This pathologic finding suggested that these tumors were derived from a gastrointestinal type mucinous epithelial component of the teratoma. Typical GI mucinous tumors 
stain for CK 20 with focal or absence of staining for CK 7 markers. Primary non-teratomatous ovarian mucinous tumors stain for CK 7 with focal or absence of staining for CK 20. Interestingly, teratomatous ovarian mucinous tumors associated with PMP have been found to stain for CK 20 similar to their lower GI counterparts, hence supporting the above findings.

In contrast to these cases, ruptured non-teratomatous ovarian mucinous tumors may result in a localized accumulation of extracellular mucin that is invariably devoid of mucinous epithelium and associated with an inflammatory infiltrate, containing histiocytes and giant cells. Multiple reports have not shown these tumors to be associated with development of PMP [12-14,16]. These tumors can be differentiated from their teratomatous counterparts due to evenly distributed cystic structures lined by proliferative mucinous epithelium, with minimal pseudomyxoma ovarii and no involvement of the ovarian surface.

In support of the findings of Ronnett, McKenney et al., evaluated the morphologic, immunohistochemical and clinical features of 42 patients with mucinous epithelial tumors of the ovary arising in association with mature ovarian teratomas [17]. The age range of these patients was 17-66 years and tumor size ranged from 5.5 to more than $200 \mathrm{~cm}$. Using the 2003 WHO classification description which breaks down the mucinous tumors based on histology types, 17 (40\%) were mucinous cystadenomas, 16 (38\%) were intestinal type mucinous epithelial neoplasm of low malignant potential (IM-LMP), 4 (10\%) were IEC and $5(12 \%)$ were invasive carcinomas. Of the 42 cases, 10 (24\%) had classic PMP and 2 (5\%) had more localized accumulation of peritoneal mucin. Evaluation of all 12 cases revealed 6 with acellular mucin, 3 with low grade mucinous epithelium (all with IM-LMP) and 3 with high grade mucinous carcinomatosis (all with ovarian mucinous adenocarcinoma). No appendiceal lesions were identified. Histologic analysis of the primary tumor showed a variable epithelial lining for mucinous cystadenomas while the IM-LMP, IEC and invasive carcinomas had a consistent lower GI histology. On immunehistochemical analysis, IM-LMP, IEC and invasive carcinomas showed strong CK 20 expression with equal expression of CK 7 and CK 20 in mucinous cystadenomas. This enteric immnuophenotype pattern confirmed the findings of previous investigators, thus concluding that ovarian mucinous neoplasms may occur in association with mature ovarian teratomas and a significant proportion of these neoplasms present with PMP, particularly the IM-LMP and mucinous carcinoma subtypes.

Another series of 44 ovarian mucinous tumors in association with mature teratomas was studied by Vang et al. [18]. All tumors displayed a gastrointestinal-type mucinous differentiation. 21 were associated with pseudomyxoma ovarii and 3 of these had PMP. Mirroring the data published by McKenney et al., cystadenomatous tumors (15 in number) without pseudomyxoma ovarii had variable expression of CK7 and CK20. All proliferative tumors without pseudomyxoma ovarii (8) expressed CK 7 with or without CK 20 and those with pseudomyxoma ovarii ( 9 cystadenomas and 5 borderline) were $\mathrm{CK}$ 7-/CK $20^{+}$. All carcinomatous tumors had pseudomyxoma ovarii, with three being $\mathrm{CK} 7^{+}$and the remaining CK 20+. Therefore they concluded that presence of pseudomyxoma ovarii was significantly associated with CK $7-/ C K 20+$ profile ( $86 \%$ with pseudomyxoma ovarii vs $13 \%$ without) and corroborated findings from other studies that, while secondary ovarian involvement should be considered in such cases, a true primary ovarian mucinous tumor of teratomatous origin can be considered as an alternative diagnosis, failing the identification of a non-ovarian source of the mucinous tumor.

Taking preliminary data into consideration, the prognosis of PMP in this setting seems to differ from that seen in the more common appendiceal primaries. Most studies however have had little to no follow up. The longest follow up was by Lee and Scully, who reported on 3 cases with PMP and showed no evidence of recurrence at 57 months, 5 years and 16 years [14]. However all three cases had no documented mucinous epithelium in the peritoneum (bland ascites). In the study by McKenney et al., no known intra-abdominal recurrences occurred in the three mucinous neoplasms with IM-LMP histology and peritoneal low grade mucinous epithelium at intervals of 23, 27 and 61 months. However mucinous carcinomas had metastases in 3 out of 5 patients and 1 documented death at 6 months after initial diagnosis. It is yet, therefore, to be determined if the presence of DPAM/PMCA in this subset of patients has the same survival as the more common appendiceal and colonic tumors.

In summary, mucinous epithelial neoplasms associated with ovarian mature teratomas exhibit a histologic and immune-phenotypic spectrum. While the IM-LMP/borderline and carcinoma subtypes resemble mucinous tumors arising in the lower GI tract, the cystadenoma subtype exhibits a non-enteric phenotype. Such resemblance to a GI tumor may be mistaken for a metastasis if the teratomatous component is not identified on histologic examination. The presence of a normal appendix after complete microscopic examination however, in a majority of cases with and without PMP, provides a compelling argument against an appendiceal primary. Ovarian teratoma associated benign and IM-LMP mucinous neoplasms with peritoneal low grade mucinous epithelium, seem to be at low risk for intra-abdominal recurrence, however numbers are few and follow up is limited. At present, teratomatous ovarian mucinous tumors represent the only cases of ovarian origin of PMP and much is still to be learned about survival and patterns of recurrence in comparison to their lower GI counterparts.

\section{References}

1. Smeenk RM, van Velthuysen ML, Verwaal VJ, Zoetmulder FA (2008) Appendiceal neoplasms and Pseudomyxoma peritonei: a population based study. Eur J Surg Oncol 34: 196-201.

2. Bree de E, Witkamp AJ, Zoetmulder FA (2000) Perioperative hyperthermic intraperitoneal chemotherapy (HIPEC) for advanced gastric cancer. Eur J Surg Oncol 26: 630-632.

3. Smeenk RM, Bex A, Verwaal VJ, Horenblas S, Zoetmulder FA (2006) Pseudomyxoma peritonei and the urinary tract: involvement and treatment related complications. J Surg Oncol 93: 20-23.

4. Ronnett BM, Kurman RJ, Zahn CM, Shmookler BM, Jablonski KA et al. (1995) Pseudomyxoma peritonei in women: a clinicopathologic analysis of 30 cases with emphasis on site of origin, prognosis, and relationship to ovarian mucinous tumors of low malignant potential. Hum Pathol 26: 509-524.

5. Ronnett BM, Yan H, Kurman RJ, Shmookler BM, Wu L, et al. (2001) Patients with Pseudomyxoma peritonei associated with disseminated peritoneal adenomucinosis have a significantly more favorable prognosis than patients with peritoneal mucinous carcinomatosis. Cancer 92: 85-91.

6. Ronnett BM, Zahn CM, Kurman RJ, Kass ME, Sugarbaker PH, et al. (1995) Disseminated peritoneal adenomucinosis and peritoneal mucinous carcinomatosis. A clinicopathologic analysis of 109 cases with emphasis on distinguishing pathologic features, site of origin, prognosis, and relationship to "Pseudomyxoma peritonei". Am J Surg Pathol 19: 1390-1408. 
Citation: Chiruvella A, Staley CA, Khanna N, Russell M, Maithel SK, et al. (2016) Pseudomyxoma Peritonei from a Borderline Mucinous Tumor Arising in an Ovarian Mature Cystic Teratoma: A Rare Case Report. Arch Surg Oncol 2: 114. doi:10.4172/2471-2671.1000114

Page 4 of 4

7. Cuatrecasas M, Guiu MX, Prat J (1996) Synchronous mucinous tumors of the appendix and the ovary associated with Pseudomyxoma peritonei. A clinicopathologic study of six cases with comparative analysis of c-Ki-ras mutations. Am J Surg Pathol 20: 739-746.

8. Guerrieri C, Franlund B, Boeryd B (1995) Expression of cytokeratin 7 in simultaneous mucinous tumors of the ovary and appendix. Mod Pathol 8: 573-576.

9. Prayson RA, Hart WR, Petras RE (1994) Pseudomyxoma peritonei. A clinicopathologic study of 19 cases with emphasis on site of origin and nature of associated ovarian tumors. Am J Surg Pathol 18: 591-603.

10. Ronnett BM, Shmookler BM, Diener-West M, Sugarbaker PH, Kurman RJ (1997) Immunohistochemical evidence supporting the appendiceal origin of Pseudomyxoma peritonei in women. Int J Gynecol Pathol 16: 1-9.

11. Young RH, Gilks CB, Scully RE (1991) Mucinous tumors of the appendix associated with mucinous tumors of the ovary and Pseudomyxoma peritonei. A clinicopathological analysis of 22 cases supporting an origin in the appendix. Am J Surg Pathol 15: 415-429.

12. Hart WR, Norris HJ (1973) Borderline and malignant mucinous tumors of the ovary. Histologic criteria and clinical behavior. Cancer 31: 1031-1045.

13. Hopkins MP, Kumar NB, Morley GW (1987) An assessment of pathologic features and treatment modalities in ovarian tumors of low malignant potential. Obstet Gynecol 70: 923-929.
14. Lee KR, Scully RE (2000) Mucinous tumors of the ovary: a clinicopathologic study of 196 borderline tumors (of intestinal type) and carcinomas, including an evaluation of 11 cases with 'Pseudomyxoma peritonei'. Am J Surg Pathol 24: 1447-1464.

15. Ronnett BM, Seidman JD (2003) Mucinous tumors arising in ovarian mature cystic teratomas: relationship to the clinical syndrome of Pseudomyxoma peritonei. Am J Surg Pathol 27: 650-657.

16. Guerrieri C, Högberg T, Wingren S, Fristedt S, Simonsen E, et al. (1994) Mucinous borderline and malignant tumors of the ovary. A clinicopathologic and DNA ploidy study of 92 cases. Cancer 74: 2329-2340.

17. McKenney JK, Soslow RA, Longacre TA (2008) Ovarian mature teratomas with mucinous epithelial neoplasms: morphologic heterogeneity and association with Pseudomyxoma peritonei. Am J Surg Pathol 32: 645-655.

18. Vang R, Gown AM, Zhao C, Barry TS, Isacson C, et al. (2007) Ovarian mucinous tumors associated with mature cystic teratomas: morphologic and immunohistochemical analysis identifies a subset of potential teratomatous origin that shares features of lower gastrointestinal tract mucinous tumors more commonly encountered as secondary tumors in the ovary. Am J Surg Pathol 31: 854-869. 